\title{
APUNTES DE METODOLOGÍA PAULINA A PARTIR DE 1 CORINTIOS 9
}

longum iter est per praecepta, breve et efficax per exempla SÉNECA, Epístolas Morales 6.5

El estudio de la historia social de los orígenes del cristianismo a partir de los escritos paulinos ha vivido una eclosión sin precedentes en las últimas décadas. La 1 Corintios se ha constituido en fuente principal de esta empresa. Esta carta ha ofrecido, por ejemplo, las principales pruebas para la teoría de la estratificación social elaborada por Malherbe, Theissen y Meeks, y criticada por Meggitt. ${ }^{1}$ Además, en la 1 Corintios aparecen textos preciosos para conocer la interacción entre los primeros creyentes en Cristo y la sociedad helenística: los juicios entre cristianos

1 A partir del estudio sobre los datos sociales extraídos de la 1 Corintios, los estudios de G. Theissen, Estudios de sociología del cristianismo primitivo, BEB 51, Salamanca - 1985 y W. A. MeEks, Los primeros cristianos urbanos. El mundo social del apóstol Pablo, BEB 64, Salamanca - 1988, 93-129, entre otros, han fijado el así llamado "nuevo consenso": las comunidades cristianas primitivas estarían internamente estratificadas. Los creyentes procederían de diversos estratos sociales: desde los esclavos, pasando por artesanos y comerciantes, hasta ciertos patrones con casas para acoger a los otros e, incluso, cargos sociales como el administrador Erasto (Rom 16,23). J. J. MegGitT, Paul, Poverty and Survival, SNTWE, Edinburgh - 1998, ha roto este consenso y ha propuesto que todos serían pobres y de extracción social baja. La discusión ha dado lugar al interesante número 84/1 de JSNT (2001), con artículos de Theissen, Meggitt, Martin y Friesen sobre la cuestión. No nos interesa tanto la discusión en sí cuanto el hecho de que la 1 Corintios haya sido la fuente principal de datos para esta investigación. 
en tribunales paganos (1 Cor 6,1-11), las diferentes ópticas respecto de las carnes sacrificadas a los ídolos $(8,1-11,1)$, etc.

Indudablemente los estudios sensibles al tenor social del cristianismo primitivo están aportando intuiciones muy interesantes a la exégesis actual. A mi modo de ver, sin embargo, algunos de ellos tienen poco en cuenta el espesor retórico y persuasivo de los textos y dan por ciertos datos básicos para sus análisis que pueden ser cuestionables desde un punto de vista retórico. Pretendo argumentar, pues, que es conveniente leer a Pablo en primer lugar con el instrumental de la crítica retórica. Puesto que las cartas son productos literarios y persuasivos, es indicado analizarlas con instrumental literario y retórico.

\section{Principios orientadores de la crítica retórica paulina}

Aunque excede a la extensión de este artículo la exposición pormenorizada sobre los fundamentos y procedimientos del acercamiento retórico propongo, no obstante, algunos principios orientadores: ${ }^{2}$

- Los textos paulinos poseen dinámica y relieve, no son planos. No todos los enunciados desempeñan la misma función: unos operan como tesis a probar, otros justifican dichas tesis, otros las amplifican o las recapitulan. Además es importante identificar la posición en la que cada enunciado es ubicado, pues una posición diferente confiere matices diversos a un mismo aserto. Por ello, es conveniente estudiar los criterios de composición del texto para identificar la función de cada enunciado y esclarecer su articulación y progresión argumentativa. Dicho estudio permite entender qué es lo que Pablo pretende mostrar y, a partir de ello, con qué pruebas lo muestra (inventio) y en qué orden (dispositio).

- Pero la crítica retórica no tiene por qué circunscribirse al texto y su articulación interna, puede -y quizás debe- ser también un método

2 Entre otros, sigo en estas reflexiones a J. MurPHY-O'CONNOR, Paul the LetterWriter. His World, His Options, His Skills, Collegeville - 1995; J.-N. AleTt।, "La rhétorique paulinienne. Construction et communication d'une pensée", Paul, une théologie en construction (ed. A. Dettwiler - J.-D. Kaestli - D. Marguerat), MoBi 51, Genève - 2004 y M. M. MitcheLL, "Rhetorical and New Literary Criticism", The Oxford Handbook of Biblical Studies (ed. J. W. Rogerson - J. M. Lieu), Oxford - 2006, 615-633. 
histórico-crítico, ya que los modelos compositivos antiguos pueden ayudar a comprender mejor los textos paulinos. Tres tradiciones son especialmente recomendables: el estudio de la teoría antigua sobre la escritura epistolar y la práctica real de las cartas; ${ }^{3}$ el estudio de la exégesis judía del tiempo -sobre todo a la hora de interpretar las citas paulinas del AT-; y la investigación sobre la retórica helenística. Entiendo, por tanto, la crítica retórica en un sentido amplio ya que comprende también la epistolaridad y la exégesis judía.

- La tercera tradición, la retórica helenística, es especialmente interesante para comprender los escritos de Pablo, ya que sus cartas, enviadas a asamblea de ambiente helenístico, poseen una evidente y pretendida intención persuasiva. Pablo usa los modelos persuasivos de su época para convencer a sus oyentes. Ahora bien, algunos estudiosos han reducido la exégesis retórica de las cartas a la identificación de la dispositio -la organización del discurso a partir de los manuales antiguos, sobre todo de Quintiliano-. Esto ha llevado, en ocasiones, a forzar los textos, ya que se intentaba por todos los medios hacer coincidir dispositio clásica con dispositio paulina. Esta práctica ha desprestigiado el método. Proponemos un procedimiento menos mecánico y más reflexivo. Entendemos la crítica retórica no sólo como el estudio y la aplicación de una colección de fórmulas y técnicas, sino sobre todo como una sensibilidad que intenta entender a Pablo desde el humus persuasivo de su época. Para adquirir esta sensibilidad es necesario profundizar en la cultura greco-romana antigua. ${ }^{4}$ Ello implica

${ }^{3}$ Cf. el estupendo manual de H. KLAUCK, Ancient Letters and the New Testament. A Guide to Context and Exegesis, Waco - 2006, con un buen compendio de cartas antiguas que puede servir para estudiar las paulinas. Para los aspectos más prácticos, cf. E. R. RIChARDS, Paul and First-Century Letter Writing. Secretaries, Composition and Collection, Downers Grove - 2004.

${ }^{4}$ M. M. MitchelL, "Rhetorical Handbooks in Service of Biblical Exegesis: Eustathius of Antioch takes origen back to School", The New Testament and Early Christian Literature in Greco-Roman Context. Studies in Honor of David E. Aune (ed. J. Fotopoulos), NT.S 122, Leiden/Boston - 2006, 350: "In my judgment this [studying Paul] is best done when we meet paideia with paideia (ours with theirs), rather than taking their techne out of its setting in paideia to use in some cookie cutter way to dissect New Testament documents. Conceived in these terms, 'rhetoric criticism' should be viewed as less a method to be applied than a sensibility to be cultivated". 
conocer y estudiar no sólo los antiguos tratados de retórica sino también discursos, cartas, ensayos y demás escritos antiguos que hacen posible acceder a la misma enciclopedia cultural que compartió Pablo.

Siguiendo el consejo de Séneca con el que iniciábamos el artículo -longum iter est per praecepta, breve et efficax per exempla-, proponemos un ejemplo práctico que puede ilustrar la importancia del análisis retórico en la exégesis paulina. Este ejemplo estriba en el uso de algunos antídotos periautológicos en 1 Cor 9. Aunque podría haber escogido otros ejemplos más claros, he preferido elegir uno polémico y un tanto novedoso para hacer más interesante la presentación.

\section{Hipótesis sobre la historia social del cristianismo primitivo según 1 Corintios 9}

1 Cor 9 ha sido un texto muy controvertido en la exégesis paulina. Entre dos capítulos exhortativos dedicados a las carnes sacrificadas a los ídolos (cap. 8 y 10), Pablo cambia de temática y habla de sí (cap. 9): de su renuncia al sustento, de su esclavitud voluntaria, de su adaptación a todos y de su lucha denodada por no ser descalificado. Muchos estudiosos han encontrado en esta tensión una prueba para cortar el texto en diferentes cartas. ${ }^{5}$ Otros, basados en 1 Cor 9,3 ("esta es mi defensa contra los que me investigan"), han estudiado el capítulo como una apología real de Pablo contra sus adversarios, y han partido de este pasaje para elaborar una reconstrucción de la situación de la 1 Corintios en la que la comunidad cuestionaría la autoridad del apóstol. En esta corriente

${ }^{5}$ El paso brusco de la temática de los idolotitos $(8,1-13)$ a la apología $(9,3)$ fue aducido como una de las pruebas para partir los cap. 8 y 9 en dos ( $c f$. resumen de hipótesis en H. MerKLEIN, "Die Einheitlichkeit der ersten Korintherbriefes", ZNW 75 [1984] 154-156). K.-K. YEO, Rhetorical Interaction in 1 Corinthians 8 and 10. A Formal Analysis with Preliminary Suggestions for a Chinesse, Cross-Cultural Hermeneutic, Biblical Interpretation Series 9, Leiden - 1995, 76-83, defiende que el cap. 9 debe ser separado en tres cartas (a la carta B: 9,24-10,22; a la C: 8,1-13+9,19-23+10,23-11; a

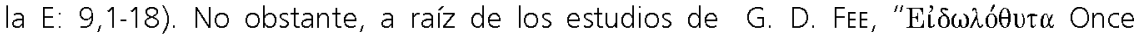
Again: An Interpretation of 1 Corinthians 8-10", Bib 61 (1980) 172-197; MerkLEIN, "Einheitlichkeit", 153-183; y, entre otros muchos, M. M. MITCHELL, Paul and the Rhetoric of Reconciliation. An Exegetical Investigation of the Language and Composition of 1 Corinthians, HUT 28, Tübingen - 1991, la unidad de 1 Cor 8,1-11,1 ha ido ganando paulatinamente en aceptación. 
interpretativa se sitúan algunos estudios relevantes sobre la historia social del cristianismo primitivo.

G. Theissen, en sus ya famosos artículos de los años 70 sobre la 1 Corintios que desencadenaron muchas discusiones que siguen candentes en la exégesis actual, propuso que en el cristianismo primitivo se desarrollaron dos tipos de predicadores: (i) carismáticos itinerantes palestinos que eran mantenidos siguiendo el mandato del Señor (cf. 1 Cor 9,14; Lc 10,7; Mt 10,10; $1 \mathrm{Tm} 5,18$ ); y (ii) organizadores de comunidades como Bernabé y Pablo que trabajaban para evitar que los criticaran como aprovechados en el contexto helenístico de los filósofos populares. La hipótesis es interesante y su explicación desde los factores políticos, económicos y culturales, muy esclarecedora. ${ }^{6}$ Theissen parte del dato de que 1 Cor 9 refleja la existencia de un conflicto actual entre estos dos grupos. En este texto, Pablo se defendería de las críticas vertidas por los misioneros palestinos a su apostolado basadas en que infringía un mandato del Señor. Nuestro análisis tratará de mostrar que es difícil deducir este conflicto a partir del pasaje. Posteriormente, P. Marshall propuso que los potentados de Corintio consideraron un insulto el rechazo de Pablo a sus ayudas a partir del estudio sobre las convenciones sociales antiguas sobre la amistad y la enemistad social. Por ello, en 1 Cor 9,1-18 el apóstol justificaría su actuación. Además, en 1 Cor 9,19-23, Pablo se defendería de la acusación de adulador oportunista. ${ }^{7}$

${ }^{6}$ Cf. G. THEISSEN, "Legitimation und Lebensunterhalt: Ein Beitrag zur Soziologie urschristlicher Missionare", NTS 21 (1975) 192-221 (traducción en ĺD., Estudios de sociología, 151-187).

${ }^{7}$ Cf. P. Marshall, Enmity in Corinth. Social Conventions in Paul's Relations with the Corinthians, WUNT II/23, Tübingen - 1987. En esta línea se sitúan también los autores que han interpretado 1 Cor 9 a la luz del contexto romano del patronazgo. Pablo rechazaría las mercedes de los patrones corintios -y se defendería en este texto de sus críticas- para evitar quedar atado por las relaciones sociales de dependencia en su predicación. Cf. C. Forbes, "Comparison, Self-Praise and Irony. Paul's Boasting and the Conventions of Hellenistic Rhetoric", NTS 32 (1986) 14-15; G. D. FEE, The First Epistle to the Corinthians, NICNT, Grand Rapids - 1987, 415-416, ejemplo eminente de lectura apologética de 1 Cor 9; P. Richardson, "Temples, Altars, and Living from the Gospel (1 Cor. 9.12b-18)", Gospel in Paul. Studies on Corinthians, Galatians, and Romans for Richard N. Longenecker (ed. L. A. Jervis - P. Richardson), JSNT.S 108, Sheffield - 1994, 94-95; D. B. MARTIN, The Corinthian Body, New Haven/London 1995, 85; C.E. GLAD, Paul and Philodemus. Adaptability in Epicurean and Early Christian Psychagogy, NT.S 81, Leiden/New York/Köln - 1995, 269 (264-272); D. G. HoRrelL, The Social Ethos of the Corinthians Correspondence. Interests and ldeology from 1 Corinthians to 1 Clement, SNTW, Edinburgh - 1996, 213-214; U. PoPLUTz, Athlet des Evangeliums. Eine motivgeschichtliche Studie zur Wettkampfmetaphorik bei 
Recientemente Goulder ha sostenido que la oposición contra Pablo en Corinto fue sustancialmente la misma que en Galacia. ${ }^{8}$ Sin embargo, él no termina de ofrecer argumentos de peso para probar que la Torah está detrás del discurso del logos de la cruz y de la sabiduría en 1 Cor 1-2. Además, los textos de la 1 Corintios son demasiado lacónicos para avalar la existencia de este grupo opositor. Podríamos citar otros muchos estudios de exégetas que han partido del supuesto dato cierto de la apología actual de Pablo en 1 Cor 9 para elaborar diversas hipótesis sobre la descripción social del cristianismo primitivo. Basten estos anteriores como muestra. $^{9}$

\section{Pruebas a favor de la lectura ejemplar de 1 Corintios 9}

Pero ¿es verdaderamente un dato seguro que 1 Cor 9 es una apología actual de Pablo? Varias pruebas de índole retórica invitan a cuestionar dicha hipótesis apologética, a favor de la lectura ejemplar: ${ }^{10}$

a) Contexto literario: 1 Cor 8,1-11,1. La lectura apologética implica una cesura con el contexto. Pablo insertaría una defensa personal

Paulus, HBS 43, Freiburg i.B. - 2004, 284-285. En este sentido, J. K. CHow, Patronage and Power. A Study of Social Networks in Corinth, ISNT.S 75, Sheffield - 1992, 102 (107-112), concluye: "When Paul wrote 1 Corinthians, he had been challenged by some rich and powerful patrons in the church partly on the grounds that he did not look like an apostle since he worked to support himself and refused to accept financial support from the church, that is, from them".

${ }^{8}$ Cf. M. D. Goulder, Paul and the Competing Mission in Corinth, Library of Pauline Studies, Peabody - 2001, 91. Esta teoría sigue, con algunas variaciones, la ya propuesta en el siglo XIX por F. C. BAUR, "Die Christuspartei in der korinthischen Gemeinde, der Gegensatz des paulinischen und petrinischen Christentums in der ältesten Kirche, der Apostel Petrus in Rom", Historisch-kritische Untersuchungen zum Neuen Testament. Mit einer Einführung von Ernst Käsemann, Ausgewählte Werke 1, Stuttgart - 1963, 1-146.

${ }^{9}$ Muy interesante es el estudio de B. W. WINTER, Philo and Paul among the Sophist. Alexandria and Corinthian Responses to a Julio-Claudian Movement, Grand Rapids $2002^{2}$, que interpreta la 1 Corintios a partir del contexto antiguo de la segunda sofística y que, como las investigaciones anteriores, también parte del dato de la apología actual paulina de 1 Cor 9 .

${ }^{10} \mathrm{Cf}$. W. WILLIS, "An Apostolic Apologia? The Form and Function of 1 Corinthians 9", JSNT 24 (1985) 33-48. La lectura exclusivamente ejemplar predomina en los autores más sensibles al tenor retórico de los textos: cf. MITCHELL, Reconciliation, 243247 y A. J. Malmerbe, "Determinism and Free Will in Paul: The Argument of 1 Corinthians 8 and $9^{\prime \prime}$, Paul in his Hellenistic Context (ed. T. Engberg-Pedersen), SNTW, Edinburgh - 1994, 239. 
(cap. 9) entre dos exhortaciones sobre las carnes sacrificadas a los ídolos (cap. 8 y 10). Esta sucesión en la que una defensa personal aparecería inserta entre dos exhortaciones carece de lógica argumentativa. En cambio, la lectura ejemplar respeta mejor el desarrollo del discurso: Pablo ilumina su primera exhortación sobre los idolotitos (cap. 8) con un ejemplo positivo, la renuncia a sus derechos (cap. 9), y otro negativo, el pecado de Israel en el desierto (10,1-13).

Concretamente, la analogía entre el cap. 8 y el cap. 9 funciona del siguiente modo: como Pablo ha renunciado a su derecho para no poner un obstáculo al avance del evangelio $(9,12$ : exousía, egkopế), así los fuertes de Corinto deben renunciar a su derecho para no causar el tropiezo del hermano débil por quien Cristo ha muerto (8,9-13: exousía, próskomma, skandalizō).

\begin{tabular}{|ll|ll|l|}
\hline Pablo & no poner obstáculo & $=>$ & al evangelio \\
\hline Corintios & n & no escandalizar & $=>$ & al hermano (por quien murió Cristo) \\
\hline
\end{tabular}

Asimismo, en la conclusión de la unidad sobre los idolotitos, la peroratio de 1 Cor 10,33-11,1, ningún término apologético es recapitulado, mientras que Pablo sí acaba con el explícitamente ejemplar: "sed mis imitadores" $(11,1)$.

b) Progresión retórica de l Cor 9. La lectura ejemplar es confirmada por la progresión de las ideas al interior de 1 Cor 9. Si algunos corintios pretenden una gnosis que les da derecho (exousia: 8,9 ) a comer, ¿cómo su apóstol no es libre para ello? ${ }^{11}$ Por eso, Pablo inicia su ejemplo en el v.1 desde este tema: sí, él es libre y apóstol. Pero dichos términos van a ser progresivamente redefinidos. Ciertamente él es un apóstol libre, pero de un modo inaudito y paradójico. Pablo se propone como un apóstol que declina sus derechos en bien del progreso del evangelio $(9,1-18)$ y como un libre que se hace esclavo para salvar a la

${ }^{11}$ C. JACON, La sagesse du discours. Analyse rhétorique et épistolaire de 1 Corinthiens, Actes et Recherches, Genève - 2006, 242: "Il sait que ce qu'il dit ne manquera pas de susciter des murmures à Corinthe: 'Ton attitude révéle que tu n'es pas encore vraiment libre!' Paul anticipe le jugement des Corinthiens et leur demande: 'Ouk eimi eleútheros;'. C'est une anticipatio rhétorique". 
mayor parte (9,19-23). Así, el v.1 anuncia todo el desarrollo discursivo en forma de partitio (presentación de temas) invertida:

\begin{tabular}{|ll|ll|}
\hline a & v.1a & ouk eimi eleutheros; \\
\hline b & v.1b & ouk eimi apóstolos; \\
\hline B & vv.1c-18 & apostol- (3x) $\quad$ y sus relativos exousía (6x); euaggel- (8x) \\
\hline A & v. 19-24 & eleútheros ón $\quad$ y sus antitéticos doulóo, doulagōgéō \\
\hline
\end{tabular}

La renuncia cada vez es mayor. Pablo dice hasta el v.18 que él no ha aprovechado su derecho a ser mantenido. Pero aunque no lo ha usado, todavía lo tiene a su disposición si lo necesita. En el v.19, va más allá. No sólo ha declinado este derecho sino que ha renunciado incluso a su libertad, fuente de todo derecho, ya que el esclavo no tiene derecho alguno. Además, no sólo ha renunciado en sentido negativo sino que se ha conformado positivamente a la identidad de otros (se ha hecho judío con los judíos, sin ley con los sin ley, etc.) Y todo ello orientado a la salvación de la mayor parte. ${ }^{12}$ La imagen atlética (vv.24-27) aplica el desarrollo anterior a la actuación de los corintios. Como los atletas, así los corintios deben privarse de todo (pánta egkrateúetai) para alcanzar la victoria. El colorido escatológico de la imagen radicaliza la exhortación. Pablo no sólo les dice que imiten su renuncia, sino que si siguen comiendo las carnes contribuyendo así a la perdición del hermano, pueden perder la corona imperecedera. La defensa del v.3 se ha ido esfumando paulatinamente. Al final de la unidad (vv.24-27), Pablo sigue aduciendo su ejemplo -corre, golpea, esclaviza su cuerpo- pero no hay atisbo de que responda a crítica alguna.

c) ¿De qué se defiende? Ha sido propuesto que él se defendería de dos cargos. Primero, en los vv.1-18, se defendería ante los que dudaban de su apostolado porque no recibía subsidio de la comunidad ${ }^{13}$-que no acogiera el salario sería un indicio de que realmente no era apóstol-. Pero

${ }^{12}$ Obsérvese la progresión de los w.15-18 centrados en motivar la renuncia ( $5 \mathrm{x}$ gár) a los w. 19-23, que subrayan la finalidad de su actuar (7x hína).

${ }^{13}$ Cf. Marshall, Enmity, 245-247, 284 y G. Barbaglio, La Prima Lettera ai Corinzi. Introduzione, versione, commento, Scritti delle origini cristiane 7, Bologna - 1995, 422. 
esta hipótesis no concuerda con la dinámica del texto: (i) que él sea apóstol es presentado como premisa (v.1), no como conclusión; (ii) las pruebas de los vv.4-12a.13-14 justifican que él tiene derecho a ser mantenido - cosa que no es criticada, según los que defienden la apología-, no el porqué de su renuncia. En segundo lugar (vv.19-23), se defendería de la crítica de adulador oportunista. Pablo se justificaría diciendo que él se ha identificado con los diferentes grupos no en beneficio personal sino para ganar a la mayor parte. Pero estas dos críticas -no es apóstol porque no cobra y es un adulador oportunista- no están relacionadas entre sí; mientras que la progresión ejemplar-de la renuncia a su derecho a la renuncia a su libertad-conecta mucho mejor los dos pasajes con propósito ejemplar.

d) 1 Cor 9 no debe ser interpretado a la luz de 2 Cor 10-13. La lectura apologética corre el riesgo de explicar anacrónicamente 1 Cor 9 a partir de 2 Cor $10-13 .^{14}$

En virtud de estas razones, parece poco adecuado leer 1 Cor 9 como una apología. Muchos han sugerido una vía media: $:^{15}$ Pablo mataría dos pájaros de un tiro, aprovecharía su ejemplo para consolidar su autoridad. Esta solución, con ser más apropiada que la anterior, debe encarar un grave escollo: ¿cómo podrían los corintios imitar precisamente lo que criticaban? Si ya cuestionaban su renuncia a ser mantenido, sería una locura por parte de Pablo proponer esta actitud conflictiva como ejemplo a emular. Así pues, con 1 Cor 9 el apóstol no pretende también defenderse sino solamente presentar un modelo a la imitación de los corintios.

${ }^{14}$ Cf. la crítica de W. Schrage, Der erste Brief an die Korinther, EKK VII/2, Düsseldorf/Zürich - 1995, II, 282.

${ }^{15}$ Esta es la hipótesis más secundada actualmente. Cf. R. LIONG-Seng PHuA, Idolatry and Authority. A Study of 1 Corinthians 8.1-11.1 in the Light of the Jewish Diaspora, JSNT.S 299, London/New York - 2005, 185-193. Entre los que realizan una lectura sensible al tenor social del cristianismo primitivo, cf. R. F. Hock, The Social Context of Paul's Ministry. Tentmaking and Apostleship, Philadelphia - 1980, 60-61 y Horrell, Socia/ Ethos, 144, 205-206. 


\section{Apología, periautología y ejemplo}

No obstante, que el v.3 no defina el punto de 1 Cor 9 no implica que deba ser ignorado. Proponemos que el tenor apologético del pasaje cumple una función subordinada al ejemplo. Pablo no pretende en 1 Cor 9 primariamente defenderse, sino que usa el lenguaje apologético para presentar con benevolencia su ejemplo personal. Para aclarar esta cuestión es de gran utilidad estudiar la forma retórica de la periautología. ${ }^{16}$ Pablo va a hablar bien sobre sí mismo ya que va a proponer que imiten su renuncia. Este elogio personal se denominó periautología. Plutarco, moralista griego una generación más joven que Pablo, estudió esta forma retórica y analizó sus consecuencias éticas en un tratadito que se titulaba: "De cómo alabarse sin despertar envidia". ${ }^{17}$

En principio, estaba mal visto que el propio orador hablara bien de sí. ${ }^{18}$ De ahí que en 2 Cor 11-12, el apóstol repite a modo de estribillo que su gloriarse es un disparate. La periautología, de por sí impertinente, era lícita en determinadas circunstancias que fueron identificadas por Plutarco: cuando no se buscaba la gloria personal (539E) sino el provecho de los demás (545D-546A); cuando el político proponía "ejemplos (paradeigmata) cercanos y familiares a los jóvenes" (544E), y otros motivos por el estilo. El de Queronea recoge una serie de antídotos que hacían aceptable la periautología (fármaka tês periautologías, 544C):

${ }^{16}$ El estudio de 1 Cor 9 a partir de la periautología ha sido apuntado por B. DODD, Paul's Paradigmatic «». Personal Example as Literary Strategy, ISNT.S 177, Sheffield 1999, 103-106. Ya el Crisóstomo dice sobre 1 Cor 9: "Y viendo que era necesario elogiarse a sí mismo -pues así podría corregir a los corintios- pero sin querer hablar grandes cosas sobre sí, observa cómo ha usado de ambos [sentimientos] para la situación requerida; alabándose a sí mismo no cuánto él se conocía, sino cuánto requería la cuestión presente" (Hom. 21 in 1 Cor; ; [PG 61, 169] traducción mía).

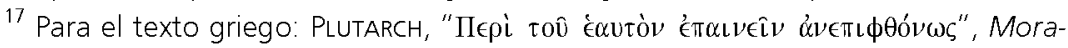
lia 539-547, LCL - Cambridge/London - 1969, 114-167; para la traducción castellana: PLUTARCO, Obras morales y de costumbres, Moralia VIII, BCG 219, Madrid - 1996, 81107. Tomo los textos de las demás obras clásicas de las mismas colecciones: el griego de la Loeb Classical Library (LCL) y el castellano de la Biblioteca Clásica Gredos (BCG). Cf. el comentario de H. D. Betz, "De Laude Ipsius (Moralia 593A-547F)", Plutarch's Ethical Writing and Early Christian Literature, SCHNT 4, Leiden - 1978, 367-393.

18 Plutarco, Mor. 539D: "El elogio proveniente de otros es lo más dulce de oír, como ha dicho Jenofonte, pero para los otros el que hace uno de sí mismo es lo más penoso". Cf. ArIstoteles, Rh. 2.6.1 (1384a); QuintILIANo, Inst. 11.1.22, etc. Cf. L. Pernot, "Periautologia. Problémes et méthodes de l'éloge de soi-même dans la tradition éthique et rhétorique gréco-romaine", REG 111 (1998) 101-124. 
desplazar el elogio propio ya sea a la audiencia (542BC), ya a la fortuna, ya a un dios (542E-543A); mezclarlo con algunos defectos personales $(543 \mathrm{~F}-544 \mathrm{C})$, etc.

Una de las circunstancias comunes de periautologías lícitas consistía en la defensa ante adversarios (540C-541A). Si uno era falsamente acusado, entonces uno podía y hasta debía hablar bien de sí, sin temor a censuras. ${ }^{19}$ En ocasiones, esta circunstancia también se convirtió en antídoto. Isócrates en su Antídosis aporta un ejemplo esclarecedor. Él pretendía reivindicar su labor educativa. Para ello realizaría un discurso que explicara sus ideas y dejara un buen recuerdo (§ 15). A tal efecto, aprovechó un proceso judicial que ya había tenido lugar y, de hecho, había perdido. Así, usó el esquema de una apología, defensa ficticia ante un tribunal, para realizar una periautología, un elogio de sí:

\footnotetext{
"si intentaba hacer mi autoalabanza (epainên emautón) no sería capaz de abarcar todo cuanto me había propuesto explicar ni tampoco podría hablar de ello de una manera atractiva e intachable. En cambio, si suponía (hypotheimēn) que se trataba de un proceso y que corría un riesgo, que el denunciante y el que me ocasionaba dificultades era un calumniador y que aquél utilizaría las calumnias pronunciadas en el proceso de cambio de fortunas, y yo pronunciaría mis palabras con el esquema de una defensa (en apologias sjémati), así sí que podría desarrollar mucho mejor todo lo que quiero" (8). "Era necesario advertir esto. Ahora, leed esta defensa que se supone escrita para un juicio (tè̀n apologian tèn prospoiouménēn mèn peri kriseōs gégrafthai) que quiere desvelar la verdad sobre mí..." (13). ${ }^{20}$
}

También Pablo habla de sus logros como apóstol (los corintios son la prueba de su apostolado) y enuncia sus derechos -lo que podría ser

19 Paradigma de periautología es el Pro Corona de Demóstenes (LCL II, 8-229; BCG 35, 367-519). En este discurso, el orador ateniense muestra su patriotismo y se ve obligado a hablar bien de sí, para defenderse de las críticas de Esquines que negaba la pertinencia de que se le concediera una corona que reconociera sus servicios públicos.

${ }^{20}$ El griego de LCL II, 185-365; el castellano de BCG 29, 80-81 (cambio "sicofanta" por "calumniador"). Pernot, "Periautologia", 115 comenta este antídoto: "En se détournant de l'auditoire, les orateurs présentent la periautologia sous la forme d'une apostrophe à l'adversaire, pour marquer qu'ils n'entendent pas entretenir le public de pareils sujets et qu'ils ne se louent que pour répondre aux imputations de la partie adverse". Cf. también G. A. KenNeDY, Classical Rhetoric and its Christian and Secular Tradition. From Ancient to Modern Times, Chapel Hill - 1980, 134: "Most great oratory and rhetorical writing has been against something even when it has been for something". 
acogido como una actitud presuntuosa- a modo de apología ante unos enemigos indeterminados que niegan su autoridad. Los adversarios están tan desdibujados que es imposible identificarlos. Así, la apología realiza una función retórica-pastoral, no personal. La defensa no es fin en sí misma, sino que le sirve al apóstol para introducir de modo adecuado su ejemplo personal. O sea, Pablo no escribe 1 Cor 9 para justificarse ante los que lo critican, sino para presentar de forma persuasiva su ejemplo personal ante su audiencia.

Él usa otros antídotos en 1 Cor 9 para que no lo tachen de presuntuoso. Estos antídotos periautológicos son modulados a partir de su experiencia de encuentro con el Mesías crucificado:

- En 1 Cor 9,2, él dice que los corintios son su obra, sí, pero una obra "en el Señor", lo que evita toda jactancia. También en Flp 3, el elogio personal de Pablo antes de su llamada (circuncidado al octavo día, hebreo de hebreos, en la ley irreprochable) es transferido a Cristo ("lo que era para mí ganancia, lo he juzgado una pérdida a causa de Cristo", Flp 3,7) ${ }^{21}$

- En 1 Cor 9,15, Pablo dice que nadie le arrebatará su gloria. Pero esta gloria consiste en algo paradójico: trabajar para no poner obstáculo al evangelio, más aún, hacerse voluntariamente esclavo. Nadie en la época vería la esclavitud como un motivo del que gloriarse. ${ }^{22}$ Así también en 2 Cor 11-12, Pablo se gloriará de sus debilidades. ${ }^{23}$

- En fin, si en l Cor 9,24-25 ha sugerido que está en juego la salvación de los corintios, en el v.27 también tiene en cuenta la posibilidad de su descalificación (adókimos), más trágica que la de

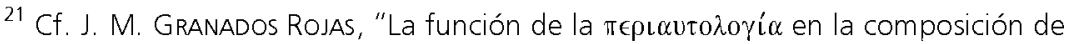
la Carta a los Filipenses", A la luz y al sentido de la Palabra. Homenaje al P. Pedro Ortiz Validiveso, S. J. (ed. J. A. Noratto Gutiérrez), Teología hoy 54, Bogotá - 2006, 269-281 y F. BIANCHINI, L'elogio di se in Cristo. L'utilizzo della "periautologia" nel contesto di Filippesi 3,1-4,1, AnBib 164, Roma - 2006.

22 "Nulla servitus turpior est quam voluntaria": "No existe esclavitud más deshonrosa que la voluntaria". SenECA, Epístolas Morales, 47; LCL I, 310; BCG 92, 280.

${ }^{23}$ Cf. A. PITTA, "Il "discorso del pazzo" o periautologia immoderata? Analisi retorico-letteraria di 2 Cor 11,1-12,18", Bib 87 (2006) 493-510. El Crisóstomo también lee 2 Cor 10-13 como una periautología, cf. M. M. MitcheLl, "A Patristic Perspective on

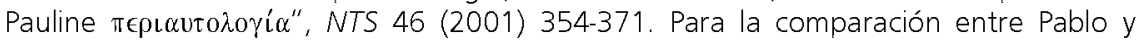
Plutarco a propósito de este texto, cf. M. WoJCIECHOWSKI "Paul and Plutarch on Boasting", JGRChJ 3 (2006) 99-109. 
ellos ya que les ha predicado (állois kērýxas). Pablo comparte con ellos la misma carrera, tampoco él ha llegado todavía a la meta (cf. Flp 3,14) ${ }^{24}$

En síntesis, ciertamente Pablo ha hablado bien de sí, pero con ello no ha pretendido jactarse sino ofrecer un ejemplo útil al discernimiento de la comunidad. Además ha subrayado sus debilidades: él también necesita esforzarse en dominar su cuerpo y no está exento de la tan temida descalificación. ${ }^{25}$ Establecemos, pues, una jerarquía de los modelos retóricos que Pablo usa en 1 Cor 9:

\begin{tabular}{|c|c|c|}
\hline modelo retórico & en función de & objetivo \\
\hline apología & periautología & introducirla con benevolencia => \\
\hline periautología & ejemplo & modelo de renuncia y acomodación => \\
\hline ejemplo & $\begin{array}{c}\text { deliberación } \\
\text { sobre losidolotitos }\end{array}$ & $\begin{array}{l}\text { que los "sabios" de Corinto renuncien a su } \\
\text { derecho en favor del hermano más débil. }\end{array}$ \\
\hline
\end{tabular}

${ }^{24}$ Pablo usa también la imagen atlética en la periautología de Flp 3. Tanto en 1 Cor 9,27 como en Flp 3,12-14, él dice que sigue corriendo y todavía no ha alcanzado la meta, para evitar aparentar que ya ha alcanzado la perfección. Cf. J.-N. AlETTI, Saint Paul Épitre aux Philippiens. Introduction, traduction et commentaire, EB 55, Paris 2005, 252.

${ }^{25}$ Quizás el énfasis en la lucha también sea un recurso periautológico. Dice Plutarco que una técnica para hacer la periautología aceptable consiste en subrayar los esfuerzos y riesgos (pônōn pollôn kai kindýnōn) arrostrados, ya que afirmar que los méritos han sido alcanzados fácilmente crea envidia (544D; cf. también Cic. De or. 2.52.209-210). De modo análogo Pablo se muestra como uno que encara grandes luchas - esclaviza su cuerpo y lo golpea!- para no ser descalificado. Además, uno de los casos más típicos de periautología es precisamente el topos del púgil. De hecho, Plutarco lo emplea dos veces en su breve tratado (541C; 543F). 


\section{Conclusión}

El estudio retórico de 1 Cor 9 invita a subordinar la lectura apologética a la función ejemplar del texto en su unidad mayor y también ha posibilitado identificar el rol de los elementos apologéticos del pasaje. Con ellos, Pablo no realiza una defensa real de su persona sino que son un procedimiento retórico al servicio de su argumentación persuasiva. Esta conclusión cuestiona la certidumbre del dato del que parten muchas investigaciones sobre la historia social del cristianismo primitivo anteriormente expuestas.

Este ejemplo exegético muestra la conveniencia de analizar los textos paulinos primariamente desde su índole retórica y persuasiva. Los estudios sociales, psicológicos y culturales pueden aportar intuiciones muy interesantes para entender a Pablo y reconstruir la vida de los primeros cristianos, pero es conveniente fijar los datos de los que partir tras un consistente análisis retórico de cada texto. La investigación retórica es una herramienta muy útil a la hora de afinar las conclusiones de la empresa exegética.

Alvaro PEREIRA DELGado

SEVILLA - ESPAÑA apereiradelgado@yahoo.es 